Spin Physics (SPIN2014)

International Journal of Modern Physics: Conference Series

Vol. 40 (2016) 1660092 (8 pages)

(C) The Author(s)

DOI: $10.1142 /$ S2010194516600922

\title{
Storage Ring Based EDM Search - Achievements and Goals
}

\author{
Andreas Lehrach \\ on behalf of the JEDI Collaboration \\ Institut für Kernphysik, Forschungszentrum Jülich, \\ Leo-Brandt Str., Jülich, 52428, Germany \\ III. Physikalisches Institut B, RWTH Aachen, \\ Otto-Blumenthal-Str., Aachen, 52074, Germany \\ a.lehrach@fz-juelich.de
}

Published 29 February 2016

\begin{abstract}
This paper summarizes the experimental achievements of the JEDI (Jülich Electric Dipole moment Investigations) Collaboration to exploit and demonstrate the feasibility of charged particle Electric Dipole Moment searches with storage rings at the Cooler Synchrotron COSY of the Forschungszentrum Jülich. Recent experimental results, design and optimization of critical accelerator elements, progress in beam and spin tracking, and future goals of the R \& D program at COSY are presented.
\end{abstract}

Keywords: Electic Dipole Moments; Storage Rings; Spin Dynamics.

PACS numbers: 13.40.Em, 11.30.Er, 29.20.Dh, 29.27.Hj

\section{Introduction}

Permanent Electric Dipole Moments (EDM) of fundamental particles violate both time invariance $T$ and parity $P$. Assuming the $C P T$ theorem this implies $C P$ violation. The Standard Model (SM) predicts non-vanishing EDMs, their magnitudes, however, are expected to be unobservably small with current experimental techniques. Hence, the discovery of a non-zero EDM would be a signal for new physics and could explain the matter-antimatter asymmetry observed in our Universe. Different approaches to measure EDMs of charged particles are pursued at Brookhaven National Laboratory (BNL) and Forschungszentrum Jülich with an ultimate goal to reach a sensitivity of $10^{-29} \mathrm{e} \cdot \mathrm{cm}$ in a dedicated storage ring. ${ }^{1,2} \mathrm{BNL}$ is pursuing the proposed proton EDM with a purely electrostatic bending field whereas the feasibility of an All-in-One lattice design with combined electrostatic and magnetic field deflectors is investigated at Jülich to also perform a deuteron or helium-3 EDM

This is an Open Access article published by World Scientific Publishing Company. It is distributed under the terms of the Creative Commons Attribution 3.0 (CC-BY) License. Further distribution of this work is permitted, provided the original work is properly cited. 


\section{A. Lehrach}

experiment, which is complimentary to the proton EDM measurement. The JEDI Collaboration has been formed to exploit and demonstrate the feasibility of such a measurement and to perform the necessary $\mathrm{R} \& \mathrm{D}$ work towards the design of a dedicated storage ring. ${ }^{3}$ As a first step R \& D work at COSY is pursued. Subsequently, an EDM measurement of a charged particle will be performed at COSY with limited sensitivity, and, on a longer time scale, the design and construction of a dedicated storage ring will be carried out. ${ }^{4}$

\section{Experimental Method}

The measurement of an EDM in a storage ring is based on the following principle: The spin is precessing in the horizontal plane governed by the magnetic dipole moment (MDM). If an EDM exists, the spin vector will experience an additional torque resulting in a change of the original spin direction that creates a vertical spin component. The vertical spin component, proportional to the size of the EDM, will be measured by scattering the stored beam at an internal target and analyzing the azimuthal distribution of the scattered particles. A coherent buildup of the vertical polarization only takes place within the time the spins of the particle ensemble stays aligned with the momentum vector. Since the spin tune is a function of the betatron and synchrotron amplitudes of the particles in the six-dimensional phase space, spin decoherence is caused by beam emittance and momentum spread of the beam and leads to a gradual decrease of the polarization buildup rate in the vertical direction. To reach the anticipated statistical sensitivity of $10^{-29} \mathrm{e} \cdot \mathrm{cm}$ a Spin Coherence Time (SCT) of $1000 \mathrm{~s}$ has to be reached. The mean challenge of such kind of experiment is a very small expected vertical component of the spin excited by the EDM and the relatively large contribution by false spin rotations due the field and misalignments errors of accelerator elements.

The magnetic dipole moment $\vec{\mu}$ and electric dipole moments $\vec{d}$ are pointing in the same direction and are proportional to the particle's spin $\vec{S}$ :

$$
\vec{\mu}=g \frac{q \hbar}{2 m} \vec{S}=(1+G) \frac{q \hbar}{m} \vec{S}, \vec{d}=\eta \frac{q \hbar}{2 m c} \vec{S},
$$

where $q$ and $m$ are the charge and the mass of the particle, respectively. The three parameters $g$ the Landé-factor $G=(g-2) / 2, G$ the magnetic anomaly and $\eta$ are dimensionless and introduced by the above equations. The spin motion of a particle in an electric and magnetic field of a circular ring is governed by the Thomas-BMT equation: ${ }^{5}$

$$
\begin{gathered}
\frac{d \vec{s}}{d t}=\vec{S} \times\left(\vec{\Omega}_{M D M}+\vec{\Omega}_{E D M}\right), \\
\vec{\Omega}_{M D M}=\frac{q}{m}\left[G \vec{B}-\frac{\gamma G}{\gamma+1} \vec{\beta}(\vec{\beta} \cdot \vec{B})-\left(G-\frac{1}{\gamma^{2}-1}\right) \frac{\vec{\beta} \times \vec{E}}{c}\right], \\
\vec{\Omega}_{E D M}=\frac{\eta q}{2 m c}\left[\vec{E}-\frac{\gamma}{\gamma+1} \vec{\beta}(\vec{\beta} \cdot \vec{E})+c \vec{\beta} \times \vec{B}\right] .
\end{gathered}
$$


$\vec{S}$ in this equation denotes the spin vector in the particle rest frame in units of $\hbar$, $t$ the time in the laboratory system, $\beta$ and $\gamma$ the relativistic Lorentz factors, and $\vec{B}$ and $\vec{E}$ the magnetic and electric field in the laboratory system, respectively. The angular frequencies $\Omega_{M D M}$ and $\Omega_{E D M}$ are defined with respect to the momentum vector of the particle. Focusing of a pure magnetic ring with vertical bending field and neglecting the EDM effect, equation 2 reduces to $\Omega_{M D M}=\frac{q}{m} G \vec{B}$. Dividing this by the particle's revolution frequency $\omega_{\text {rev }}=\frac{q}{\gamma m} \vec{B}$ leads to the number of spin precession per particle revolution, referred to as spin tune $\nu_{s p}^{0}=\gamma G$, giving the number of spin revolutions relative to the momentum vector per particle revolution around the invariant spin axis for the reference particle of an ideal ring.

In a real ring with field imperfections and magnet misalignments the beam and spin motion is perturbed and will lead to closed-orbit excursions and subsequently to a change of the spin tune $\nu_{s p}$ as function of the betatron and synchrotron amplitudes of individual particles. The main systematic error of an EDM measurement in a storage ring is supposed to come from radial magnetic and vertical electric fields. They will mimic an EDM rotation via the MDM of the particle. Already a radial magnetic field in the order of $10^{-17} \mathrm{~T}$ would correspond to an EDM of $10^{-29} \mathrm{e} \cdot \mathrm{cm}$ and cannot be measured directly. By the use of two counter rotating beams a radial magnetic field will lead to a vertical separation of the two beams. Measuring this beam separation with high-precision beam position monitors (BPM) is one of the main challenges to control non-vanishing radial magnetic fields. This method only works if the beam currents and phase space distribution of the two beams are absolutely the same or are determined very precisely.

\section{Experimental Results at COSY}

For the experimental results presented here a vector polarized deuteron beam has been utilized at a momentum of $p=0.970 \mathrm{GeV} / \mathrm{c}$ with roughly $10^{9}$ stored particle per fill. After injection and acceleration of the initially vertically polarized beam an RF solenoid was used to transfer the spin polarization into the horizontal plane. To measure the polarization, the beam was slowly extracted onto a carbon target using a white noise electric field. The polarization was then measured by elastically scattered deuteron in the EDDA detector. ${ }^{6}$ The spin tune was $\gamma G \approx-0.161$ with a beam revolution frequency of $f_{\text {rev }}=752.543 \mathrm{kHz}$. The leads to a spin precession frequency in the horizontal plane of $\gamma G \cdot f_{\text {rev }} \approx 125 \mathrm{kHz}$. To determine the absolute arrival time of the events within the accelerator cycle a long range TDCs was used to time stamp the events. ${ }^{7}$

\subsection{Spin Tune Measurement}

With dedicated analysis tools the spin tune can be determined with ultra-high precision. ${ }^{7,8}$ A simple fit to the in-plane horizontal polarization precession is not possible, because on average only about one event is detected per twenty-four spin 

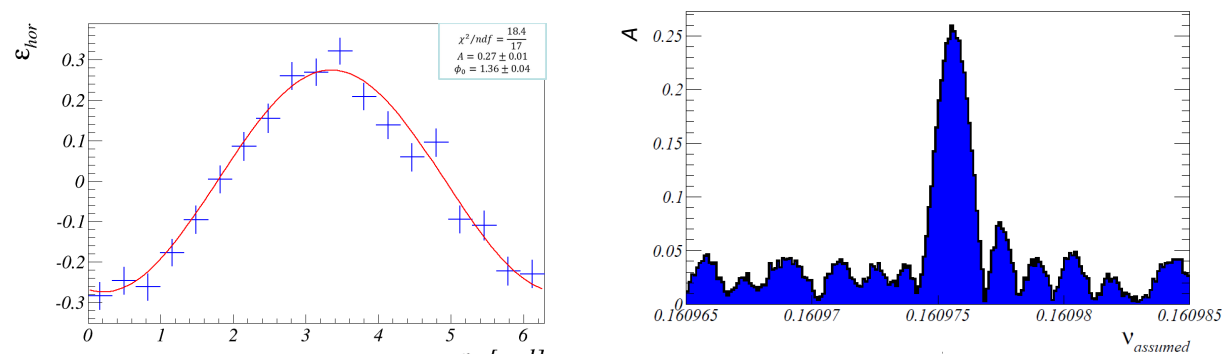

Fig. 1. Fit of the first spin oscillation period (left plot). Amplitudes $A$ of the horizontal spin precession as function of the assumed spin tune $\nu_{\text {assumed }}$ (right plot).

revolutions with an EDDA detector event rate of $5 \mathrm{kHz}$ and a spin precession frequency $120 \mathrm{kHz}$. With a rough value of the spin tune using the beam's revolution frequency all events in each time period are mapped into the first spin oscillation period for a macroscopic time interval of one or a few seconds. After that the first spin oscillation period can be fitted with a sinusoidal function (Fig. 1, left plot). This fitting procedure is repeated for several values of the spin tune $\nu_{s p}$ in a certain range. The extracted amplitude obtained from the fit are shown in Fig. 1 (right plot) as a function of the assumed spin tune $\nu_{\text {assumed }}$. The first approximation of the spin tune is than given by the spin tune obtained by the largest amplitude $\nu_{\max }\left(A_{\max }\right)$ of the horizontal spin precession. After mapping all macroscopic time intervals $\Delta t$ into the corresponding first spin oscillation period, the phase parameter of the spin is determined from the fits to distributions as shown in Fig. 2 (left plot) using $\nu_{\max }$. For a constant phase as function of the cycle time the actual spin tune is also constant and equals $\nu_{\max }$. The $1^{\text {st }}$ derivative of the fit gives the deviation of the spin tune from $\nu_{\max }$ during one cycle. Fig. 2 (right plot) shows an example for a dependence of the spin tune on the cycle time. The spin tune can be determined to a precision of $10^{-8}$ in a macroscopic time interval of $\Delta t=2 \mathrm{sec}$ Assuming a linear change of the spin tune over the time of one cycle, a determination of the average spin tune in a cycle of $100 \mathrm{sec}$ with precision of about $10^{-10}$ is possible.
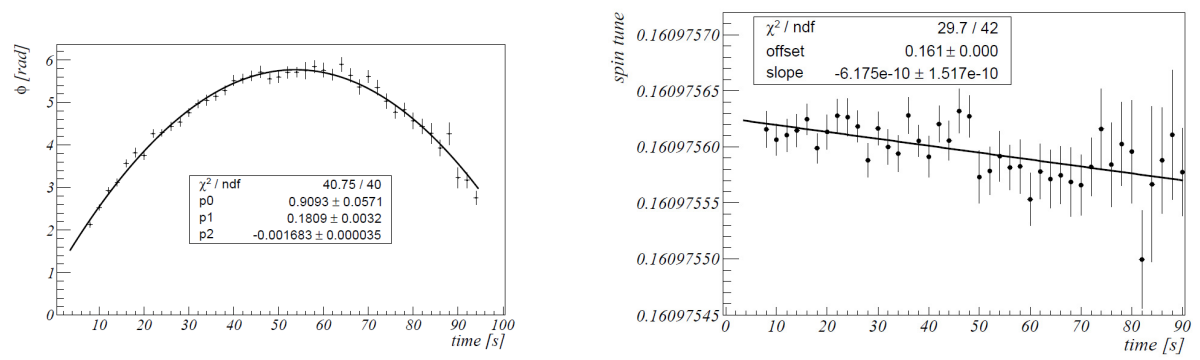

Fig. 2. Spin phase as a function of time in the cycle for $\nu_{\max }$ (left plot). Spin tune $\nu_{s p}^{0}$ versus time in cycle in $\Delta t=2 \mathrm{sec}$ time intervals (right plot). 
With this ultra-high precision spin tune measurement an extreme powerful tool is now available to study systematic effects in COSY.

\subsection{Spin Coherence Time}

Effective measures to counteract spin decoherence is space-space cooling, beam bunching and multipole correction of field errors. Especially the correction of field errors and beam chromaticity by sextupole magnets has been studied in great detail at COSY. ${ }^{9}$ To explore all degrees of freedom, studies were made with two beam preparations: (1) a beam whose horizontal emittance was expanded using white noise applied to a pair of electric field plates, and (2) a period of electron cooling followed by bunching in order to produce a spread of synchrotron amplitudes.

Initial cooling of the beam reduced contributions from other degrees of freedom. The three families of arc sextupole magnets (MXS, MXL, and MXG) at COSY were adjusted to find those settings where both the $\mathrm{X}$ and $\mathrm{Y}$ chromaticity were zero. For each of the two beam preparations, scans were made of either MXS or MXG while holding the other constant. Along each scan, measurements were made of the horizontal polarization lifetime. The SCT was found to be a function of MXG with a maximum value at $\mathrm{MXG}=11.44 \%$ of full power supply scale and a beam with enlarged synchrotron amplitude, as shown in Fig. 3 (left plot). Out of each scan, the sextupole settings for the point of largest SCT was found and plotted as a function of MXG as seen in Fig. 3 (right plot). This experiment demonstrates the possibility to achieve SCT of about $1000 \mathrm{~s}$ for polarized deuterons based on a combination of electron cooling, beam bunching, and the setting of sextupole fields near places where both the $\mathrm{X}$ and $\mathrm{Y}$ chromaticities vanish.

\section{Design and Optimization of Accelerator Elements}

\subsection{RF Wien filter}

For the first direct EDM measurement at COSY a high-power RF-E/B spin flipper is required with an electric field gradient of more than $1 \mathrm{MV} / \mathrm{m}$ and a magnetic
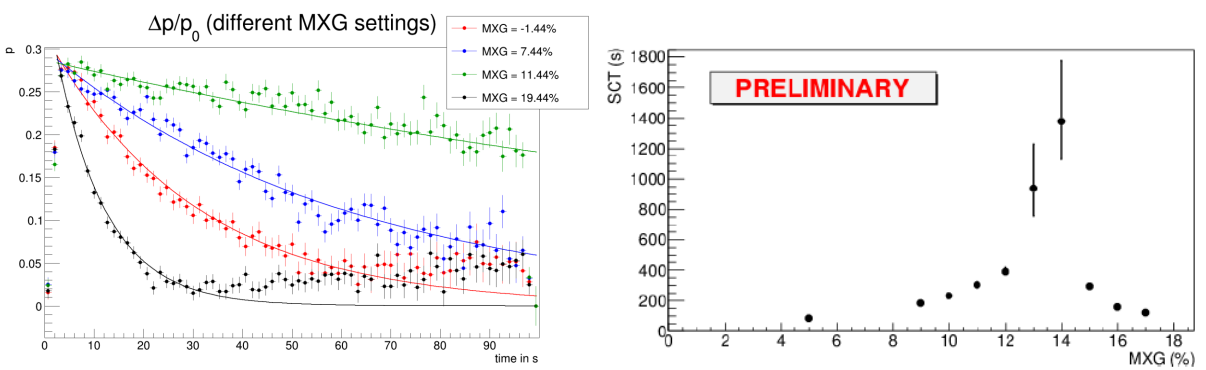

Fig. 3. Measurements of the horizontal polarization lifetime as a function of the strength of the MXG sextupole family (left plot). SCT as a function of sextupole setting MXG sextupole family with MXS set to $10 \%$ and MXL set to $1.45 \%$ of power supply full scale (right plot). 


\section{A. Lehrach}

field of roughly $70 \mathrm{G}$ in a broad frequency range of roughly 0.1 to $1 \mathrm{MHz}$. Both field types have to be exactly perpendicular to each other. When operated in the "Wien filter" mode, the Lorentz force on the beam is canceled by a certain ratio of electric and magnetic field magnitude.

A prototype RF Wien filter has been developed and successfully commissioned with lower power to carry out a feasibility test. ${ }^{10}$ It has been demonstrated experimentally, that continuously flipping the vertical polarization of a $970 \mathrm{MeV} / \mathrm{c}$ deuteron beam can be performed without exciting any coherent beam oscillation. The device was operated at the $f_{r e v}|\gamma G-1|$ harmonic of the spin precession frequency at roughly $871 \mathrm{kHz}$. Similar measurements at the $f_{\text {rev }}|\gamma G+1|$ harmonic at $630 \mathrm{kHz}$ are planned. The benefit of lower RF frequency is less damping of the induced oscillation of the vertical polarization component. By a $90^{\circ}$ rotation of the device around the beam axis, this RF E/B dipole in Wien filter mode will modulate the spin tune via the MDM. In conjunction with the fields distributed along the accelerator ring this leads to an EDM induced polarization build-up of the vertical polarization component.

A second prototype device based on a strip-line design is also developed together with the High-Frequency Institute of RWTH Aachen University.

\subsection{Electrostatic Deflector}

For the final EDM ring, high-field electrostatic deflectors have to be developed. Major development steps are the optimization of the shape of electrostatic field plates with suitable magnet coil configurations and R \& D work on surface treatments that can yield high electric field gradients. For an All-in-One EDM storage ring with a radius of $r=30 \mathrm{~m}$, transverse electric fields of roughly $17 \mathrm{MV} / \mathrm{m}$ and magnetic fields up to $1.6 \mathrm{kG}$ are required in combined electrostatic/magnetic field deflectors. Electrostatic deflectors, previously employed as beam separators in the Tevatron, have been transferred from Fermi National Accelerator Laboratory (FNAL) to Jülich to get experience on surface treatment and operation of high voltage electrostatic field plates and finally to refurnish this devices to further increase field gradients toward the requirements for the final ring.

\subsection{Beam Position Monitors}

A prototype SQUID (Superconducting QUantum Interference Device) based BPM will be developed and finally tested in the COSY ring. As a first step magnetic pick-ups operated at room temperature in a Rogowski coil configuration have been developed. The main advantage of the quartered Rogowski coil design to measure up-down and left-right positions is the response to the particle bunch frequency and the compactness of the configuration itself. The magnetic coil BPMs will be benchmarked in a laboratory test system. The calibrated BPMs will then be installed in the ANKE target chamber for beam tests. A first estimate shows that one needs 
a sensitivity in the order of $1 \mathrm{fT} / \sqrt{\mathrm{Hz}}$. SQUIDs with this ultra-high sensitivity are available but were never successfully tested in an accelerator environment. ${ }^{11}$

\section{Beam and Spin Tracking}

Full spin-tracking simulation of the entire experiment is absolutely crucial to explore the feasibility of a first direct EDM measurement at COSY and a planned dedicated EDM storage ring. For a detailed study during the storage and buildup of the EDM signal, one needs to track a large sample of particles for billions of turns. Given the complexity of the tasks, particle and spin dynamics simulation programs must be benchmarked and tracking results compared to beam experiments performed at COSY, to ensure the required accuracy of the obtained simulation results. COSY Infinity $^{12}$ and Mode $^{13}$ are utilized for this purpose, both based on map generation using differential algebra and the subsequent calculation of the spin-orbital motion for an arbitrary particle. Integrating program, solving equations of particle and spin motion in electric and magnetic fields using Runge-Kutta integration, are also been used for benchmarking.. ${ }^{14}$ They have been shown to be accurate to sub-part per billion levels in describing the muon (g-2) spin precession frequency. The integration step size is $0.5 \mathrm{ps}$, making it rather slow with a possible maximum tracking time of about $10 \mathrm{~ms}$ for a particle in the ring. This simulation programs are suitable to study effects that occur on short time scales.

The spin motion in homogenous electromagnetic fields has been investigated in case of a non-vanishing EDM. Furthermore the ability to calculate time-dependent transfer maps, which is necessary to model the influence of RF fields on beam and spin motion for the first direct EDM measurement at COSY, was implemented. Since the particle motion is perturbed by imperfections of the storage ring magnets, the existing misalignment commands for shifts, tilts and rotations can be superimposed to study randomized sets of magnet misalignments and the resulting closed orbits can be corrected by the orbit correction system to suppress false spin rotations via the MDM. ${ }^{15,16}$

\section{Future Goals of the R \& D Program}

The $\mathrm{R} \& \mathrm{D}$ program will continue with prototyping of critical accelerator elements and tests with polarized beams at COSY. Different methods to perform a first direct EDM measurement at COSY will be further investigated by spin-tracking simulations in order to quantify the systematic limits and finally perform an EDM measurement at COSY. For the design study of a dedicated EDM storage ring, lattice design in conjunction with the design of all accelerator elements will be the major task for the JEDI collaboration in the upcoming years.

\section{Acknowledgments}

The author wish to thank the COSY crew for preparing a dedicated accelerator setup and the members of the JEDI collaborations for excellent cooperation 


\section{A. Lehrach}

and fruitful discussions. This work has been financially supported by the Jülich Aachen Research Alliance JARA-FAME (Forces and Matter Experiments), the Forschungszentrum Jülich via COSY FFE and the EU Integrated Infrastructure Initiative (FP7-10 INFRASTRUCTURES-2012-1, Grant Agreement No. 227431).

\section{References}

1. V. Anastassopoulos et al., A proposal to measure the proton electric dipole moment with $10^{-29}$ e cm sensitivity, Brookhaven National Laboratory, 2011; http://www.bnl.gov/ edm/files/pdf/proton_EDM_proposal_20111027_final.pdf

2. JEDI Collaboration webpage; http://collaborations.fz-juelich.de/ikp/jedi

3. A. Lehrach, Project Overview and Computational Needs to Measure Electric Dipole Moments at Storage Rings, in it in Proc. $11^{\text {th }}$ International Computational Accelerator Physics Conference (ICAP 2012) (Rostock-Warnemünde, Germany, 2012), MOAAI1, p. 7, ISBN 978-3-95450-116-8.

4. F. Rathmann et al., The search for electric dipole moments of light ions in storage rings, J. Phys. Conf. Ser. 447, 012011 (2013).

5. L.H. Thomas, Phil. Mag. 3, 1 (1927); V. Bargmann, L. Michel and V. L. Telegdi, Phys. Rev. Lett. 2, 435 (1959).

6. D. Albers et al., Eur. Phys. J. A 7 22, 125 (2004).

7. Z. Bagdasarian et al., Phys. Rev. ST Accel. Beams 17, 052803 (2014).

8. J. Pretz, Measurement of Electric Dipole Moments at Storage Rings, in Proc. $9^{t} h$ International Conference on Nuclear Physics at Storage Rings (STORI 14) (Sankt Goar, Germany, 2014), to be published in Physica Scripta.

9. G. Guidoboni, Spin coherence time studies of a horizontally polarized deuteron beam at COSY, in Proc. $9^{\text {th }}$ International Conference on Nuclear Physics at Storage Rings (STORI 14) (Sankt Goar, Germany, 2014), to be published in Physica Scripta.

10. S. Mey, R. Gebel, A Novel RF-E $\times$ B Spin Manipulator at COSY, these proceedings.

11. W. Vodel, K. Mäkiniemi, Meas. Sci. Technol. 3, 1155 (1992); .W. Kornack, S.J. Smullin, S.-K. Lee, and M.V. Romalis, Appl. Phys. Lett. 90, 223501 (2007).

12. K. Makino, M. Berz, COSY INFINITY Version 9, Nucl. Inst. and Meth. in Phys. Res. A 558 (2005).

13. A. Ivanov, Yu. Senichev, Matrix Integration of ODEs For Spin-Orbit Dynamics Simulation, in Proc. $5^{\text {th }}$ International Particle Accelerator Conference (IPAC 14) (Dresden, Germany, 2014), MOPME011, p. 400, ISBN 978-3-95450-132-8.

14. Yu. Senichev, Storage Ring EDM Simulation: Methods and Results, in Proc. $11^{\text {th }}$ International Computational Accelerator Physics Conference (ICAP) (RostockWarnemünde, Germany, 2012), TUADI1, p. 99, ISBN 978-3-95450-116-8.

15. M. Rosenthal, Investigation of Beam and Spin Dynamics for EDM Measurements at COSY, in Proc. $9^{t} h$ International Conference on Charged Particle Optics (CPO-9) (Brno, Czech Republic, 2014), to be published in the Cambridge Journals Online Microscopy and Microanalysis.

16. S. Chekmenev, Estimation of Systematic Errors for Deuteron Electric Dipole Moment at COSY, these proceedings. 